\title{
Les enjeux de la traduction publicitaire
}

Dr. Dalia Mohamed El Sayed El Toukhy

Professeur adjoint

À

Faculté Al-Alsun-Université Ain Shams

Et

La Faculté de langues- Université Princesse

Nourah Bint Abdulrahman 



\section{Résumé}

La publicité est un aspect essentiel de la société consommatrice et en même temps un enjeu économique. C'est une forme de communication basée sur l'art de la persuasion qui a envahi les médias dans le monde entier. La mission d'un bon message publicitaire repose sur une profonde connaissance du public cible.

La traduction du message publicitaire a occupé, depuis longtemps, l'intérêt des traducteurs qui se trouvent, dans le processus de la traduction, en face de plusieurs enjeux: la fonction du texte, son effet sur le public visé et son adéquation à la culture de la société cible.

La présente étude est à priori motivé par la prédominance et l'essor de la publicité dans notre vie et l'importance accrue du rôle de la traduction dans la commercialisation mondiale.

L'étude est répartie en quatre domaines d'analyse : le premier abordera l'effet de l'adaptation dans le transfert du message publicitaire, le second, le rôle de la rhétorique dans la publicité, le troisième, sera focalisé sur l'analyse du rôle et des modalités de l'argumentation et le quatrième sera axé sur l'image publicitaire. 
Comment le traducteur peut-il transposer les figures de rhétorique de manière à servir le message publicitaire ? Dans quelle mesure est-il autorisé à expliciter l'implicite et procéder par étoffement ? Comment peut-il transposer tous les éléments argumentatifs du message source ?

C'est justement à ces questions que nous tenterons de répondre dans cette recherche.

Mots clés : Publicité-Adaptation-Traduction-Communication.

\section{تعديات الترجمة الدعائية}

تعد الدعاية عنصرًا أساسيًا من عناصر المجتمع الاستهلاكي، وشكل من أثكال التو اصل يقوم على فن الإقناع، استطاع غزو وسائل الإعلام في مختلف أنحاء

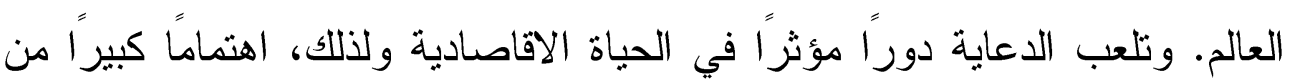
جانب شركات الإنتاج و التسويق.

وقد شغلت ترجمة الرسالة الدعائية، منذ وقت طويل، اهنمام المترجمين الذين يجدون أنفسهم، في عملية الترجمة، أمام عدة تحديات : وظيفة النص، جودة الرسالة الدعائية و أثرها على الجمهور المستهدف، ومدى ملائمته الرسالة وتو افقها مع ثقافة

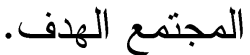

الدافع لإجر اء هذه الدر اسة يرتكز علي المكانة التي تحتلها الدعاية في حياتنا الاجتماعية والثقافية والاقتصادية، ودور الترجمة في تسويق النصوص الدعائية علي المصعيد المحلي و العالمي. 
تتكون الدراسة من أربعة مجالات للتحليل : يتتاول الأول أثز التكيف في نقل الرسالة الدعائية، والثاني دور الأسلوب البلاغي في الدعاية، ويرتكز الثالث علي تحليل دور وطرق الحجاج في الإقناع في النصوص الدعائية، أما الرابع فينطرق للصورة الدعائية ودورها في نقل الرسالة للجمهور المستهدف. تجيب هذه الدر اسة علي التساؤلات التالية : كيف يمكن للمترجم نقل الصور البلاغية من لغة إلى أخرى بحيث بحافظ من جهة، على الرسالة الدعائية، ومن جهة

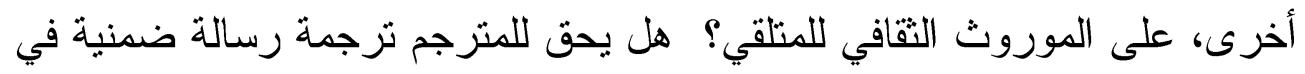
النص الدعائي؟ كيف يمكن للترجمة الدعائية نقل كل العناصر الحجاجية المتو اجدة في الرسالة الإعلانية المصدر؟ هل يحق للمترجم تفسير الصورة الدعائية إذا لم يكن يصاحبها نص مكتوب؟

هذه هي على وجه التحديد التساؤلات التي سنحاول الإجابة عليها خلال هذه الدراسة.

La publicité fait partie de notre environnement. Elle est tout autour de nous, on la voit, l'observe, l'entend par le biais de différents médias (télévision, presse, radio, internet, cinéma, etc.) Chacun peut en effet citer au moins un slogan ou une annonce publicitaire qui l'a marqué et auquel il s'est accoutumé.

La publicité n'est pas une simple distraction, mais un aspect essentiel de la société consommatrice et en même temps un enjeu économique. C'est une forme de communication basée sur l'art de la persuasion qui a envahi les médias dans le monde entier. Le discours publicitaire joue davantage un rôle 
considérable dans la propagation des produits ou des articles objet de la publicité dans les différentes cultures.

Un bon message publicitaire repose sur une profonde connaissance du public cible (les habitudes des jeunes adolescents, les intérêts des adultes, etc.) Connaître son public cible signifie s'intéresser à ses habitudes de consommation, à la façon dont il prend une décision. II s'agit de faire une analyse complète du client potentiel afin de retenir son attention et de le convaincre d'acheter un produit.

" [...] (la publicité) ne peut pas être confondue avec de I'information, au sens strict. Adaptée aux économies de marché, outil de concurrence, la publicité n'est pas pour autant exclusivement marchande et elle peut défendre des institutions, des causes sociales ou politiques. Elle peut beaucoup mais elle ne sait pas faire miracles. On recourt à elle de façon très variable selon les secteurs, la conjoncture, les saisons. "

(Lendrevie J., \& Baynast A., 2004 : 10)

Pour réaliser sa mission auprès du public visé, un message publicitaire doit contenir plusieurs éléments de persuasion comme : 
- Un titre ou un slogan qui attire l'attention tels que les procédés iconographiques (images, symboles etc.) ou les procédés graphiques (couleur, contraste etc.)

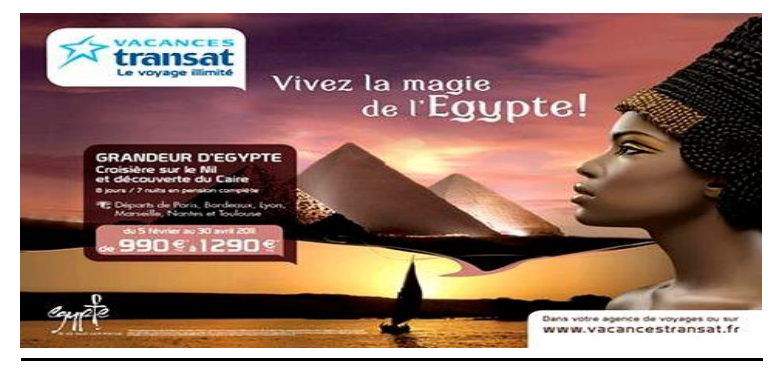

- Des mots à connotation positive. Ex. : brillant, sublime, inégalable, élégant, etc.

- Une argumentation convaincante ou un discours bref qui dit l'essentiel. Ex : Renault...la force silencieuse

- Des caractéristiques précises associées au produit qui lui donnent une valeur crédible (statistique, témoignage de clients satisfaits, etc.)

La traduction du message publicitaire a occupé, depuis longtemps, l'intérêt des traducteurs. Sur ce sujet Mathieu Guidère écrit : "En raison de ses difficultés intrinsèques, le texte publicitaire a très tôt intéressé les traducteurs et les traductologues de tous bords. Leur intérêt remonte aux années 1970, bien avant les linguistes qui ne se consacreront au langage et au discours publicitaires qu'au cours de la décennie suivante 
(Greven 1982 ; Tremblay 1982 ; Everaert- Desmet 1984. Le numéro spécial de la revue Meta publié en 1972 sous la houlette de Roger Boivineau marque le début véritable de la réflexion sur la traduction publicitaire. [...] II faut attendre le début des années 2000 pour voir paraitre la première étude d'ensemble exclusivement consacrée à la traduction publicitaire. Guidère (2000) s'appuie sur un corpus homogène d'annonces multilingues qui lui permet d'aborder les principales difficultés soulevées par la traduction des publicités françaises vers l'anglais, l'arabe et l'espagnol. A travers une série d'études de cas concrets qui propose une description exhaustive des différents niveaux d'analyse en expliquant, à chaque fois, les choix de traduction et les décisions prises par le traducteur." (Guidère, 2009, pp. 417-419)

La traduction des textes publicitaires a assisté à une évolution rapide dans les méthodes de travail employées. "Les traducteurs se sont rendu compte de l'inadéquation du critère de fidélité pour ce genre de textes, dont le contenu et le but n'avaient rien de sacral pour exiger un respect scrupuleux de la forme ni même du fond. De ce constat de décalage entre la visée du message et le type d'équivalence recherchée naît la consigne d'adaptation pour les textes publicitaires. 
L'adaptation marque un tournant salvateur dans l'exercice professionnel de la traduction publicitaire, mais elle a donné lieu à une multitude de pratiques qui oscillent entre la reformulation métaphorique et la réécriture totale du texte original.

Gràce à l'adaptation, la pratique de la traduction publicitaire entre dans une nouvelle phase ou les professionnels vont rechercher une équivalence fonctionnelle des messages publicitaires. Le traducteur [...] pense sa traduction en fonction d'un but précis, généralement un effet à produire sur le récepteur à partir d'un argumentaire particulier. " (Tatilon 1990 : 245)

Le traducteur d'un texte publicitaire se trouve donc en face de plusieurs enjeux : la fonction du texte, son effet sur le public visé et son adéquation à la culture de la société cible. Pour affronter ces enjeux, le traducteur doit posséder, comme l'indique Mathieu Guidère, un double savoir : « un savoir culturel vendeur et un savoir-faire technique susceptible de faciliter la recontextualisation du message. De cette concentration des compétences est née une nouvelle spécialité ayant pour nom la localisation publicitaire " " اطوطين الدعاية qui représente depuis quelques années la phase ultime de cette évolution de la profession. [...] La localisation publicitaire consiste à adapter une communication commerciale à un locus (province, pays, région, 
continent) en prenant en charge la totalité du processus d'adaptation textuelle et iconographique.» (Guidère 2009 : 428429)

Pour réaliser cette localisation publicitaire, le traducteur devient : "maître d'œuvre pour l'intégralité du message et responsable à la fois de la traduction du texte, des retouches éventuelles des images qui l'accompagnent, mais également de la mise en forme finale de la communication publicitaire : ajustements éventuels du texte et de l'image, choix des couleurs, adaptation des symboles, etc.

Dans les détails, cette évolution représente un saut qualitatif important qui a permis de briser le carcan de la pratique professionnelle jusque-là cantonnée dans le seul domaine linguistique et textuel. La localisation apparaît alors comme la solution d'une libération tant attendue. Persuader et localiser est donc une des caractéristiques du texte publicitaire. " (ibid :430)

La persuasion peut également être vue dans la publicité dans l'emploi des figures rhétoriques. Par exemple, l'emploi des allitérations "Pepsi nous rassemble et nous sommes toujours ensembles. » des anaphores "لاطازج الا الطازج), des assonances «Lego développe l'ego » des répétitions « Le meilleur d'ici et 
d'ailleurs au meilleur prix » de l'ironie "Mini Tuc ... ce n'est pas la taille qui compte. "

De là est née la méthode fonctionnelle ou traduction du «skopos » publicitaire. Cette méthode fait partie de la théorie sur l'action traductionnelle proposée par Holz-Mänttärri qui perçoit la traduction comme une sorte particulière d'action traductionnelle basée sur un texte de départ. Le terme skopos, d'origine grecque qui signifie but ou objectif, a été introduit pendant les années 1970 par le théoricien allemand Hans J. Vermeer comme un terme technique désignant le but du texte d'arrivée et de l'action traduisante. II s'appuie sur le principe selon lequel tout type d'action traductionnelle peut être considéré comme une action ayant un but ou une fonction et que, par conséquent, la traduction peut, elle aussi, avoir un but particulier. Dans cette méthode, le but de la traduction ou «skopos du translatum " peut être différent de celui du texte de départ. Vermeer précise que «le texte de départ et le texte d'arrivée peuvent différer considérablement l'un de l'autre, non seulement dans la formulation et la distribution du contenu, mais aussi dans leurs buts respectifs, lesquels déterminent la façon dont le contenu est arrangé ». Le skopos se concentre surtout sur le but de la traduction qui détermine les méthodes de traduction et les 
stratégies employées pour arriver à un résultat fonctionnellement adéquat. (Cf. Guidère M., Publicité et traduction, éd, L'Harmattan, Paris, 2000)

Sur le plan stylistique, la persuasion peut être exprimée à l'aide de l'interrogation "Vous n'en voulez plus de ces photocopieurs fragiles ? " des exclamations «Phytovitaminezvous les cheveux! (PHYTOVITAMINES), Minéralise-toi !!! (eau minérale SAN BERNARDINO). », de l'impératif «NESCAFE restez motive» qui donnent au message publicitaire une motivation et un style bref pour aller droit au but et exhorte le public immédiatement au vouloir d'achat.

Les questions sont surtout employées dans les gros titres des publicités. Le lecteur souhaite une réponse à cette question et pour cette raison il lira la publicité

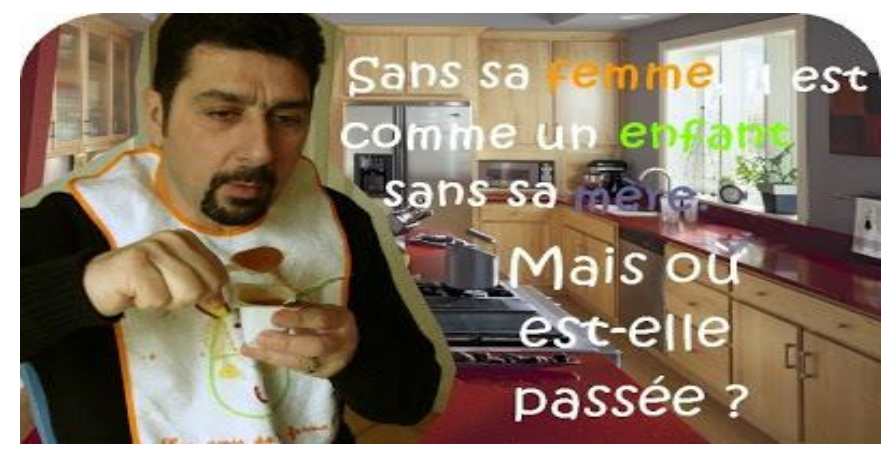


La langue publicitaire utilise aussi l'ellipse qui raccourcit les phrases en omettant certaines parties linguistiques de la phrase comme par exemple les verbes ou les pronoms personnels, ce qui rend les informations courtes et à un degré élevé de densité d'information. "Un portable - un Siemens »

La manière d'influencer et de persuader les gens est souvent réalisée par la combinaison des mots et des images. En se servant du langage publicitaire, on essaie de toucher le lecteur d'une manière émotive. Mais c'est surtout grâce aux images que les lecteurs sont touchés. Pourtant, cela dépend aussi du produit pour lequel on fait de la publicité.

Comme nous venons de mentionner, la publicité est fermement liée à la culture de chaque peuple. Raison pour laquelle la traduction doit être assez stricte et rigoureuse. Un exemple qui montre la complexité de ce type de traduction est ce qui est arrivé à la compagnie «Ford » quand elle a industrialisé sa nouvelle voiture «Pinto » en Brésil, et elle n'a réalisé aucune vente, car elle a chargé un traducteur qui maîtrise la langue portugaise mais ignore le dialecte brésilien. II a utilisé un mot qui n'est pas respectable dans le dialecte brésilien. Et avec le changement du nom de la voiture à «Corcel », il y avait un taux considérable de ventes. 
«Le transfert culturel du message publicitaire reste le défi majeur pour tout traducteur professionnel qui doit agir en tant que vrai médiateur interlinguistique et interculturel. I/ est donc nécessaire d'ausculter, avant de traduire, les pensées, les modes de vie, les motivations, les attentes des clients effectifs ou potentiels ». (Guidère, 2000 : 127)

Face à ce défi, le traducteur peut recourir à certaines techniques comme "la profilisation " (garder le fond et la forme du texte original) » (Guidère, $2000: 128$ ) C'est pour cette raison que l'adaptation fait soumettre les éléments principaux de la communication aux caractéristiques propres du marché cible.

La présente étude est à priori motivé par deux raisons : d'une part, la prédominance et l'essor de la publicité dans notre vie et l'importance accrue du rôle de la traduction dans la commercialisation mondiale.

D'autre part, nous avions eu la chance de coopérer avec l'Agence Universitaire de la Francophonie (AUF) dans la cadre du projet intitulé : «Hybridation et Traduction » pour la conception d'un module à enseigner à distance intitulé : «La traduction publicitaire ».

Dans ce module, les apprenants recevront une initiation théorico-pratique de la traduction publicitaire qui intègre de 
nouvelles technologies dans l'enseignement au niveau Master. L'objectif de ce module est de faire connaître aux apprenants les enjeux et les paramètres de la traduction et du style publicitaire, ainsi que des difficultés découlant des transferts culturels. Le projet vise également à apprendre aux étudiants un savoir-faire traductif qui les aide à traduire des textes de niveaux variés provenant de sources commerciales et publicitaires diverses en appliquant les méthodes et procédés de traduction étudiés.

La conception de ce module nous a encouragés à mettre en évidence les enjeux de la traduction publicitaire à l'aide d'une recherche qui met en relief les problèmes auxquels fait face le traducteur dans le processus du transfert du message publicitaire et les moyens utilisés pour régler ces problèmes.

Nos références de base, dans cette étude sont les ouvrages de Mathieu Guidère intitulés : «Publicité et traduction (2000) », «La Communication multilingue : traduction commerciale et institutionnelle (2008)» et l'ouvrage de Jean Michel Adam ayant pour titre : «L'argumentation publicitaire, $2005 »$

Les publicités choisies ont été sélectionnées du module appliqué, ainsi que des différents médias : internet, magazines à 
condition qu'elles soient conformes aux domaines analysés au cours de la recherche.

Ce travail sera réparti en quatre domaines d'analyse : Dans le premier nous aborderons l'effet de l'adaptation dans le transfert du message publicitaire, dans le second, le rôle de la rhétorique dans la publicité, dans le troisième, nous focaliserons l'analyse sur le rôle et les modalités de l'argumentation et le quatrième sera axé sur l'image publicitaire.

Comment le traducteur peut-il transposer les figures de rhétorique de manière à servir le message publicitaire ? Dans quelle mesure est-il autorisé à expliciter l'implicite et procéder par étoffement ? Comment peut-il transposer tous les éléments argumentatifs du message source?

C'est justement à ces questions que nous tenterons de répondre dans les pages suivantes.

\section{Publicité et adaptation}

Les cultures dans chaque pays sont différentes. Nous pouvons donc penser que cela se retrouve également dans les publicités, vu que nous n'avons pas les mêmes façons de voir les mêmes produits. Pour pouvoir réaliser une bonne publicité qui charmerait sa cible, il est important que les responsables tiennent 
compte des différences entre les cultures. Car une publicité qui fonctionne bien aux Etats-Unis, ne fonctionnera sans doute pas dans les pays arabes et vice versa. Par exemple une publicité mettant en avant la nudité sera mal vue dans les pays arabes. II est donc primordial que les managers marketing s'intéressent à la culture du pays ciblé, pour rendre efficaces leurs publicités, s'ils veulent percer sur le marché

L'adaptation publicitaire, selon Boivineau, " est une opération ardue, parfois impossible. De ce fait, elle demande à l'adapteur nombre de qualité et de connaissances, de même qu'une solide expérience. [...] Le traducteur adapteur devra mettre en ceuvre des principes éprouvés, fondés sur la psychologie appliquée et résumés par la formule magique du publicitaire : AIDA-A signifie : attirer l'attention; I: susciter l'intérêt ; $D$ : éveiller le désir ; $A$ : provoquer l'achat. " (Boivineau R., 1972 : pp.5-7)

Voyons ensemble comment l'adaptation en traduction s'étale dans la publicité de la lessive liquide "persil gel" pour les vêtements noirs : 


\begin{tabular}{|l|l|}
\hline Préserve l'intensité du noir \\
Et des couleurs foncées \\
Baume de figue noire
\end{tabular}

Devant une telle publicité, le traducteur n'a pas eu recours ni à la transliteration ni à la modulation, mais il s'agit d'une adaptation qui a produit une nouvelle publicité différente et adéquate à la culture des pays arabes où le le style vestimentaire traditionnelle des femmes « العباية " est en général en couleur noire, ce qui rend la publicité compréhensible pour le public cible.

La traduction du message publicitaire diffère donc selon l'intention du locuteur et du public ciblé. Ainsi, à travers un choix de mots et de signes visuels, la publicité oriente le message dans la langue cible de façon différente. 
Tout en mettant l'accent sur le fait que c'est un shampooing pour la Abaya شاشامبو العباية), le traducteur a mis en exergue trois qualités du détergent qui sont au centre de l'intérêt du public cible : son effet sur la poussière et les saletés « بزيل "un effet important pour la femme en Arabie vu le climat poussiéreux des pays du Golf, "حماية اللون الأسود pour l'éclat de la couleur noire de la Abaya, et son odeur qui dure longtemps 《عطر يدوم طويلًا

Tandis que la version française a focalisé le message sur deux effets : «préserve l'intensité du noir » et l'odeur «baume de figue ».

Sur le plan iconographique, nous remarquons un changement complet répondant aux coutumes des pays du Golf en affichant une femme voilée et souriante portant la Abaya, une métonymie qui symbolise la couleur noire et la satisfaction, ce qui reflète la bonne qualité de la lessive liquide "persil gel".

\section{Publicité et Rhétorique}

Toute entreprise de publicité, cherchant la sensibilisation du public cible, devrait veiller à mobiliser les connaissances du récepteur, à le stimuler et à éveiller sa pensée. Elle devrait, pour ainsi dire, mettre en œuvre certaines techniques afin d'affiner ses 
campagnes de propagande, ses produits, ou son message publicitaire.

Parmi les techniques qu'emploient ces sociétés dans leurs affiches publicitaires, figure la rhétorique publicitaire.

D'une façon générale, la rhétorique c'est l'art du discours ou plutôt l'art de convaincre. C'est en quelque sorte l'art de parler avec éloquence afin de laisser chez le récepteur certaines empreintes, et appeler le public cible à la contribution. Cet outil, du moment où il fait appel à l'imagination du récepteur, lui insinue une certaine idée, surtout que c'est lui qui fait une part de l'effort pour déchiffrer le message en question.

Selon Jacques Durand «Si la publicité a un intérêt culturel, c'est à la pureté et à la richesse de sa structure rhétorique qu'elle le doit : non pas à ce qu'elle peut apporter d'information vraie, mais à sa part de fiction ». (Durand, 1970: 75).

Il incombe ainsi au traducteur chargé du transfert d'un message publicitaire de reproduire le même effet et la même ambiance qu'a créés la publicité dans sa langue d'origine. Le traducteur peut se trouver ainsi confronté à certains défis d'ordre linguistique, stylistique ou culturel, vu les différences existantes entre la langue de départ et la langue d'arrivée, mais se voit 
également contraint de surmonter ces défis de manière à éviter d'aplatir ou neutraliser la publicité.

Nous tenterons, dans les pages suivantes, d'appréhender les difficultés relatives à la traduction de quelques figures de rhétorique dans la publicité, tout en expliquant les changements opérés pendant le transfert du message.

Dans ce domaine nous allons adopter la répartition traditionnelle selon laquelle la métaphore et la métonymie jouent sur le sens de mots ; l'oxymore et l'antithèse jouent sur la pensée. Notre étude s'étend également aux figures de construction comme l'anaphore et le chiasme.

\section{La Métaphore :}

La métaphore est une figure de rhétorique qui rapproche un comparé et un comparant "C'est un procédé par lequel le sens d'un mot spécifique est transposé à un autre à l'aide d'une analogie ou une comparaison implicite (Le Petit Larousse Illustré, 2002).

Par le biais de la métaphore, on effectue un transfert de sens, on substitue un élément par un autre qui lui ressemble ou l'évoque. "L'intérêt de la métaphore tient à sa capacité à améliorer l'évaluation du message via un effet de génération. Les 
arguments produits par soi sont plus crédibles que ceux qui sont fournis explicitement. [...] La métaphore visuelle consiste à ne pas donner explicitement l'argument proposé, en ce sens elle produit un effet de génération : l'individu exposé au message doit compléter le sens avec ses propres compétences. La métaphore fait partie des tropes (métaphore, métonymie et épiphonème) qui intensifient la pénétration de l'encodage du message persuasif. »(Capelli A., 2007 :9-10)

Nous verrons dans la publicité ci-dessous, comment cette métaphore est exprimée tant par l'image que par le texte qui y est inscrit, et nous tenterons d'en proposer une traduction, tout en analysant les procédés auxquels nous avons eu recours pour communiquer le message.

La présente publicité est relative au produit chocolatier Twix :
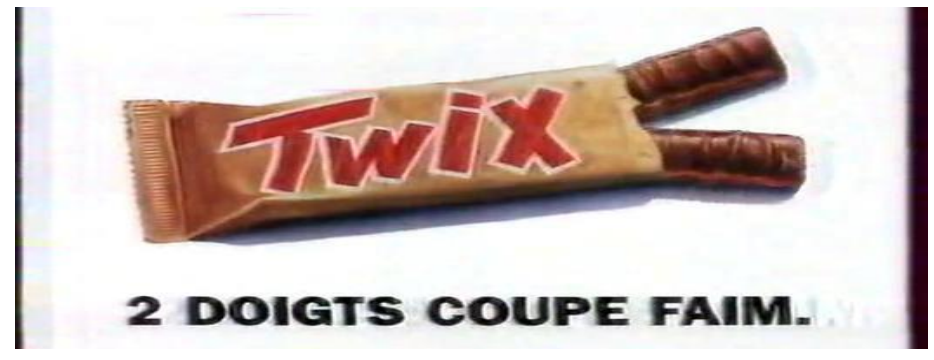

(2 doigts.... Coupe-faim.) 
Nous pouvons clairement voir que les deux doigts du chocolat prennent l'allure des deux lames du ciseau, d'où l'emploi du verbe «couper». De plus, le nom masculin composé «coupefaim » appartient au registre familier qui signifie « modérateur de l'appétit » et en arabe 《ابض للش هية 》.

\section{https://www.larousse.fr/dictionnaires/francais/coupe-faim/19815}

Dans cette publicité métaphorique, l'image réside dans l'assimilation du chocolat aux lames d'un ciseau. En analysant cette publicité, nous sommes donc censées rendre ce sens-là vers l'arabe tout en préservant l'effet de l'image, l'aspect rhétorique de la publicité et le registre de langue.

Nous aurions pu proposer pour « 2 doigts coupe-faim »:

$$
\text { صباعين يقطعوا جو عك ou صباعين يقبضو اش هينك }
$$

Si nous avons proposé ces deux expressions, c'est parce que يقبضو | restituera le lexique du découpage, et que 》 ش شيتك

Cependant, ces phrases appartiennent à la langue arabe classique, un fait qui pourrait atténuer l'effet visé par la publicité source. Pour cette raison, nous préférerons la traduire par :

$$
\text { صباعين هيخلصوا علي جوعك }
$$




\begin{tabular}{|c|c|}
\hline \hline L.D & L.A \\
\hline 2 doigts coupe-faim & صباعين هيخلصو ا على جو عك (Syntagme verbal) \\
(Syntagme nominal) & \\
\hline
\end{tabular}

Nous avons donc essayé de transposer la figure rhétorique de la langue source, vers la langue cible, tout en étant obligé de changer le champ lexical et sémantique de cette métaphore.

Cette modulation métaphorique et grammaticale, selon Hélène Chuquet et Michel Paillard, consiste à opérer un changement de point de vue en changeant le champ lexical duquel tient chaque expression. "La modulation métaphorique se définit comme un changement de point de vue. Celui-ci intervient au niveau du mot, de l'expression ou de l'énoncé pris globalement ; il relève du lexique et /ou de la grammaire. [...] le recours à des métaphores différentes d'une langue à l'autre est l'un des problèmes de traductions les plus connus. » (Chuquet \& Paillard, $1989: 26-27)$

La traduction arabe a plutôt assimilé le chocolat à des bandits ou à des assassins qui tueront notre faim et nous rassasieront. Ainsi l'angle de la vision, sous lequel ce message est délivré a totalement changé. Nous avons également effectué 
une transposition, en changeant le syntagme nominal dans (2 doigts coupe-faim) en syntagme verbal (صباعين هيخلصو ا على جو عك))

La métonymie :

Selon le Dictionnaire de Poétique et de Rhétorique, la métonymie est une «Figure par laquelle un mot désignant une réalité $A$ se substitue au mot désignant une réalité $B$, en raison d'un rapport de voisinage, de coexistence, d'interdépendance, qui unit $A$ et $B$, en fait ou dans la pensée. " (Morier, 1961: 743) Voyons comment ce procédé peut être employé dans la publicité relative à une compagnie française pour le soutien scolaire :

Dans cette publicité, nous pouvons voir de quel potentiel la publicité parle, et de quoi exactement cette société veille à s'occuper. Nous pouvons voir que juste la moitié du visage d'Einstein, présentée sur l'affiche, juxtaposée à celle d'un enfant, insinue que tout enfant peut devenir brillant comme Einstein, devenu symbole de l'intelligence.

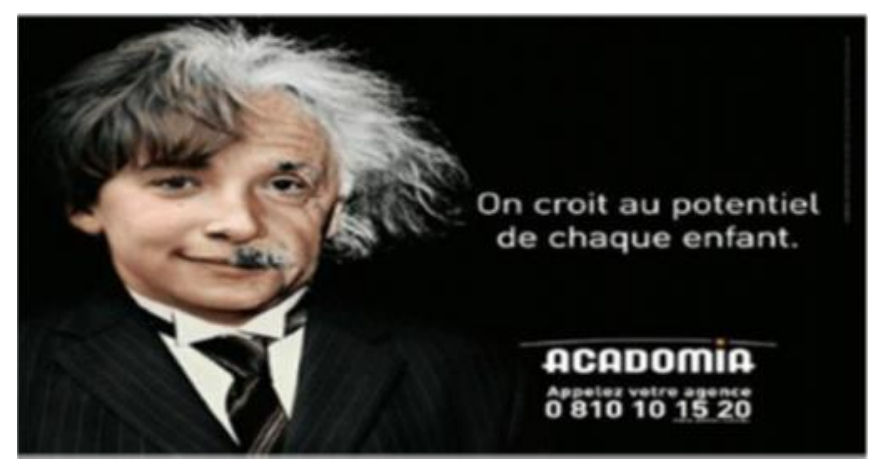


D'ailleurs la métonymie ne réside pas ici au niveau du texte, mais plutôt au niveau de l'image, car la moitié du visage d'Einstein présentée représente l'intelligence et la créativité.

Ainsi, pour traduire une telle annonce, nous pouvons très bien transmettre le sens en gardant cette partie insinuée, dans l'image, telle quelle, celle de l'intelligence et dire dans ce cas " avec une translittération. L’adjectif 《 inciterait le public cible à réfléchir et à regarder l'image pour détecter la signification de cette force potentielle.

Nous aurions pu faire autrement en élucidant un peu le sens et dire :

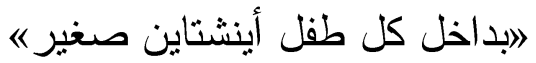

qui signifie «Chaque enfant a en soi un petit Einstein. », mais nous avons exclu cette proposition pour ne pas élucider l'implicite du message, au risque que le message ne devienne trop direct perdant ainsi son mystère, son éloquence et garde son caractère métonymique.

\section{L'Antithèse :}

L'antithèse c'est une mise en parallélisme de deux réalités opposées. En d'autres termes, c'est un procédé stylistique qui consiste à opposer, dans la même phrase, deux mots ou groupes de mots de sens contraire afin de mettre une idée en relief par 
un effet de contraste. Selon le Dictionnaire de Poétique et de Rhétorique, l'antithèse est une «Figure par laquelle on établit un contraste entre deux idées, afin que l'une mette l'autre en évidence. " (Morier, 1961 : 114)

Nous allons également essayer d'appliquer et d'analyser ce procédé rhétorique dans la publicité de Peugeot :

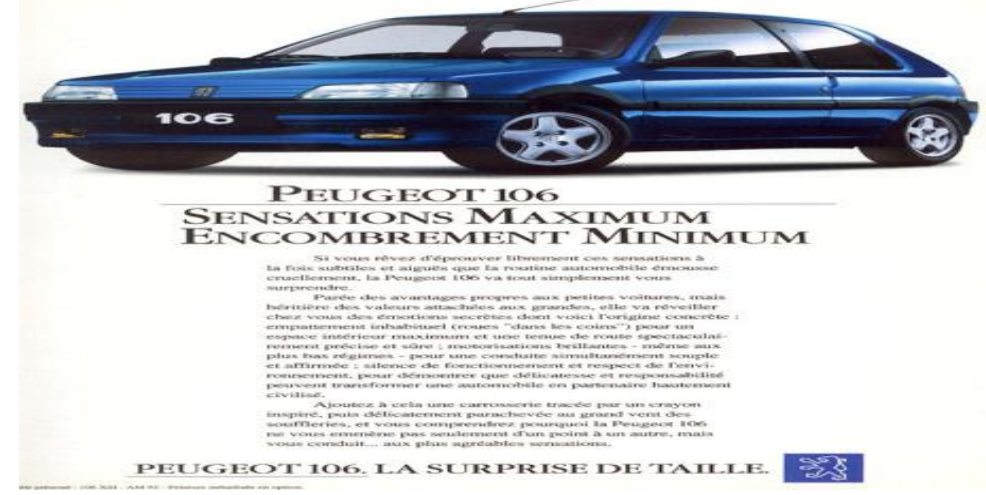

(Peugeot 106 : Sensation Maximum, Encombrement minimum.)

Nous pouvons voir que l'antithèse réside ici dans l'opposition entre maximum et minimum. Cette antithèse-là vise à mettre en valeur l'aspect confortable de cette voiture, et à mettre en exergue l'élément de la taille qui nous procurera une sensation de luxe et de bien-être.

Ainsi nous pouvons aisément rendre ce slogan, dans la langue arabe, tout en gardant l'antithèse : 


\begin{tabular}{|c|c|}
\hline L.D & L.A \\
\hline $\begin{array}{c}\text { Peugeot } 106 \\
\text { «Sensation maximum, } \\
\text { encombrement } \\
\text { minimum.» }\end{array}$ & 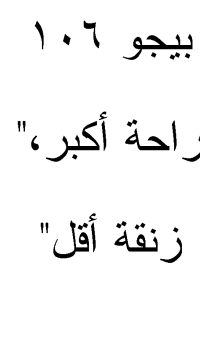 \\
\hline
\end{tabular}

Ainsi l'antithèse entre «Maximum et minimum » a été rendue par " " أكبر و أقل ", ce qui confère au message toute sa force, captant, par suite le récepteur. Toutefois la séquence de la langue source émet une musicalité provenant de la rime finale entre maximum et minimum. Or, cette rime-là a disparu dans la langue d'arrivée, au profit du message. Le terme «زنقة "زم) appartient au registre classique et signifie selon le dictionnaire en ligne Al Maany :

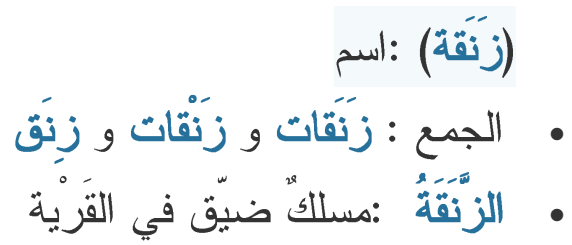

https://www.almaany.com/ar/dict/arar/\%D8\%B2\%D9\%86\%D9

$\% 82 \% \mathrm{D} 8 \% \mathrm{~A} 9 /$

Nous avons vu comment une antithèse peut surprendre ou stimuler le public cible et combien il serait facile de la transposer dans la langue d'arrivée. 
L'oxymore :

Avec l'oxymore, on associe deux mots opposés pour créer une curieuse sensation. Les contraires s'annulent, et créent une image nouvelle, originale et appréciée.

Selon le Dictionnaire de Poétique et de Rhétorique, d'Henry Morier, l'oxymore est "Une sorte d'antithèse dans laquelle on rapproche deux mots contradictoires I'un paraissant exclure logiquement l'autre. » (Morier, 1961 :828)

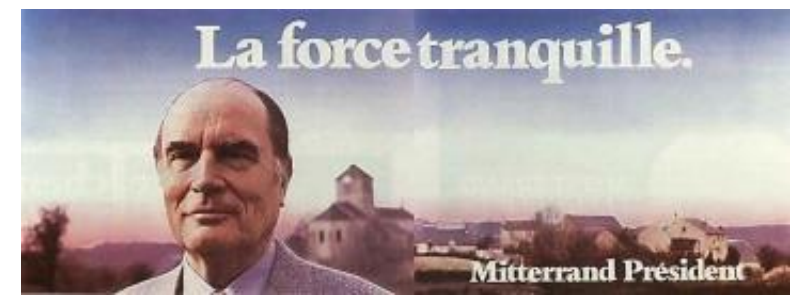

(La force tranquille)

Cette affiche fait partie de la campagne de propagande électorale de l'ex-président français François Mitterrand. Cette affiche vante les mérites du candidat en encadrant ses qualités par un paradoxe flagrant, procédé appelé oxymore.

Selon Morier, «Le rapprochement de termes antinomiques permet de recréer une impression mêlée " visant à capter le récepteur. » (Morier, 1961 : 830) 
Nous pouvons donc rendre l'oxymore dans cette expression frappante par « القوة الهادئة ", en procédant ainsi par translittération pour préserver la force et l'authenticité du message.

D'après cette analyse non exhaustive sur la modalité de traduction des publicités à caractère rhétorique, nous avons vu comment certaines figures de style concernent le sens et l'idée elle-même, comme la métonymie et la métaphore, et comment ces figures-là obligent le traducteur, par la suite, à adopter une certaine position et à être vigilent par rapport à la stylistique de la phrase.

Ainsi, ces entreprises-là, outre la rhétorique à laquelle elles ont recours pour toucher l'imagination du récepteur, ont également recours à un autre genre de publicité, qui s'appelle la publicité argumentative.

«La publicité argumentative est une forme de publicité qui s'adresse à la raison. Elle cherche à convaincre en utilisant des arguments rationnels pour renforcer le message. » (Http://www.academie-des-sciencescommerciales. org/dictionnaire_new/definition.php ? id=5953. 
C'est ainsi que ce genre de publicité est généralement utilisé dans les campagnes de sensibilisation, relative à titre d'exemple à l'hygiène, à la santé publique ou aux dangers d'un tel ou tel phénomène.

L'argumentation publicitaire vise à influencer les opinions et les comportements du récepteur à travers tout un processus argumentatif qui le mènera à adopter l'idée présentée.

Nous nous pencherons, dans les pages suivantes, sur certaines affiches de campagnes de sensibilisation, ayant le plus recours à l'argumentation, et nous analyserons les modalités d'argumentation dans ces affiches publicitaires, et les modalités de leur traduction.

Nous aurons donc affaire à la traduction de publicités employant l'argumentation par la narration ainsi que par l'information, et nous verrons comment l'argument peut dans certains cas être implicite et, dans d'autres cas, être explicite.

\section{Argumentation publicitaire narrative :}

Argumenter en narrant un récit est l'un des moyens de l'argumentation, et « Le cas le plus fréquent est celui de la simple création d'un contexte narratif dans lequel l'objet se trouve en quelque sorte mis en situation. » (Adam, 2005 : 132) 
L'affiche ci-dessous est une publicité de sensibilisation contre le tabac :

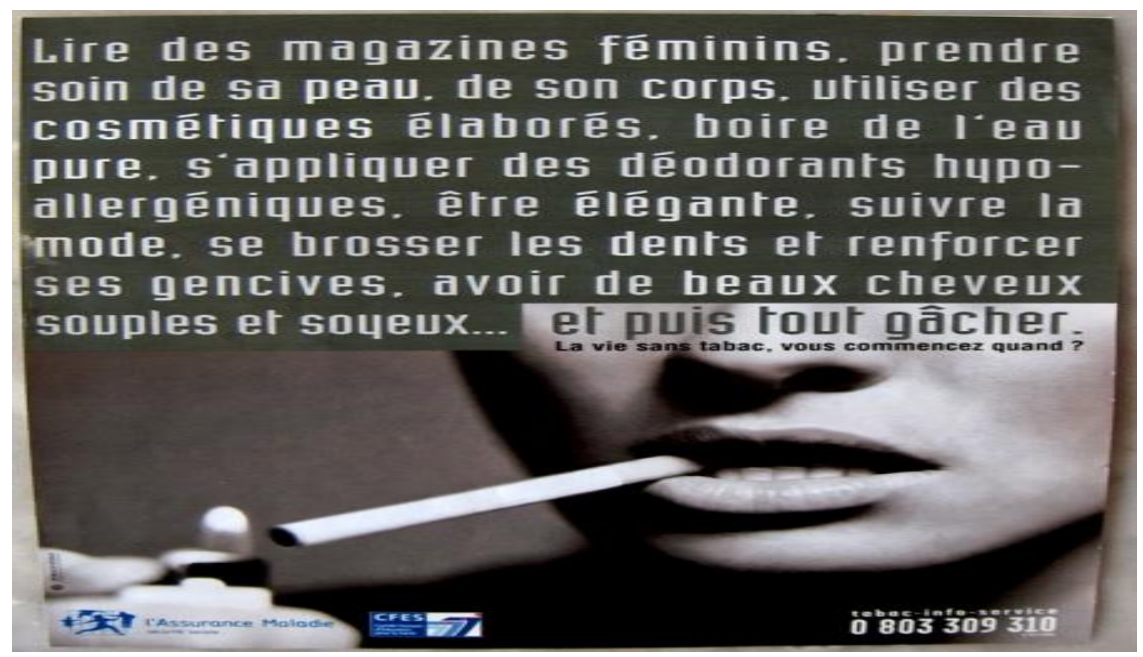

Selon Adam et Bonhomme, "L'inventio c'est la détermination du thème du discours et des arguments valables pour l'auditoire qu'il s'agit de convaincre. » (Adam, 2005 : 101)

Ainsi le thème du discours réside dans la prévention du tabac, qui peut gâcher et détruire toute une vie. II est clair que cet argument n'a pas été ouvertement mentionné. Après avoir employé le style narratif de la vie exemplaire d'une femme, l'argument "La tabac gâche la vie », a été exprimé implicitement à l'aide d'une interrogation finale : «vous commencez quand? $\gg$ 
C'est ainsi que l'on comprend que ce qui gâcherait une vie, c'est le tabac.

Nous pouvons voir que dans le texte source, la publicité s'adresse directement aux femmes à travers la moitié d'un visage féminin qui focalise d'ailleurs sur la partie qui consomme le tabac. Pourtant le texte n'emploie aucun pronom personnel, qu'à la dernière phrase de l'annonce : « vous commencez quand ? »

Nous pouvons donc traduire ce passage en explicitant le récepteur féminin, ainsi :

\begin{tabular}{|c|c|}
\hline L.D & L.A \\
\hline $\begin{array}{l}\text { Lire des magazines féminins. } \\
\text { Prendre soin de sa peau, de son } \\
\text { corps. Utiliser des cosmétiques } \\
\text { élaborés. Boire de l'eau Pure. } \\
\text { S'appliquer des déodorants } \\
\text { hypoallergéniques. } \\
\text { Étre élégante, se brosser les dents } \\
\text { et renforcer ses gencives. } \\
\text { Avoir de beaux cheveux souples et } \\
\text { soyeux... Et puis tout gâcher. } \\
\text { La vie sans tabac, vous commencez } \\
\text { quand? }\end{array}$ & 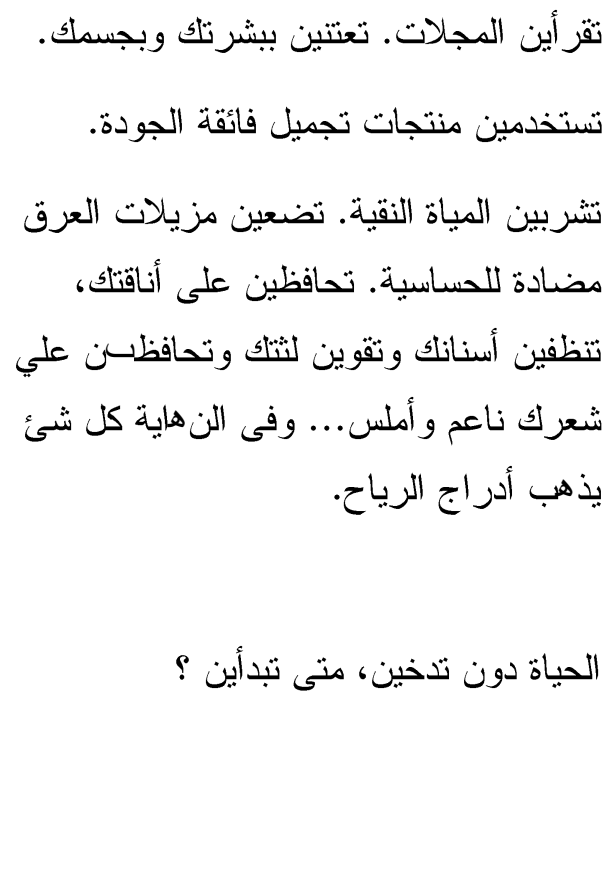 \\
\hline
\end{tabular}


Nous avons eu recours à la modulation grammaticale en conjuguant les verbes à l'infinitif du texte source

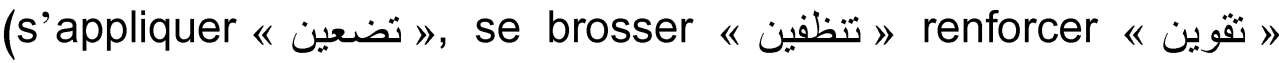

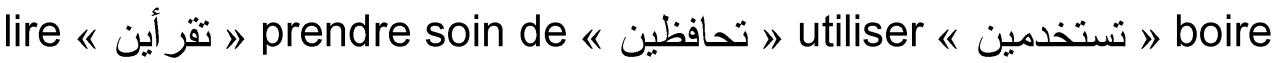
《 ( ثشربين)

Nous avons également eu recours à une transposition par étoffement métaphorique dans « تذهب أدراج الرياح ", pour « tout gâcher ». L'expression "يذهب أدراج الرياح " est une expression idiomatique arabe, signifiant que l'effort a vainement abouti et que les tentatives ont fait long feu, selon le Dictionnaire des expressions idiomatiques(arabe-arabe), de Wafaa Kamel Fayed

$$
\text { (فايد ، 2000:212) }
$$

La narration permet en effet de faire partager les valeurs en jouant sur les sentiments que peuvent susciter chez le destinataire les personnages et les événements, comme l'espoir, la crainte, l'empathie, l'identification. « Le discours narratif permet ainsi de rendre compte d'une évolution entre un début et une fin. "

(http://www.love-communication.fr/les-types-de-discours-etleurs-interet-dans-lapublicite-a86614277 . 
Nous allons voir, dans les deux affiches suivantes, comment l'argument peut être camouflé dans l'image, et non pas dans le texte :
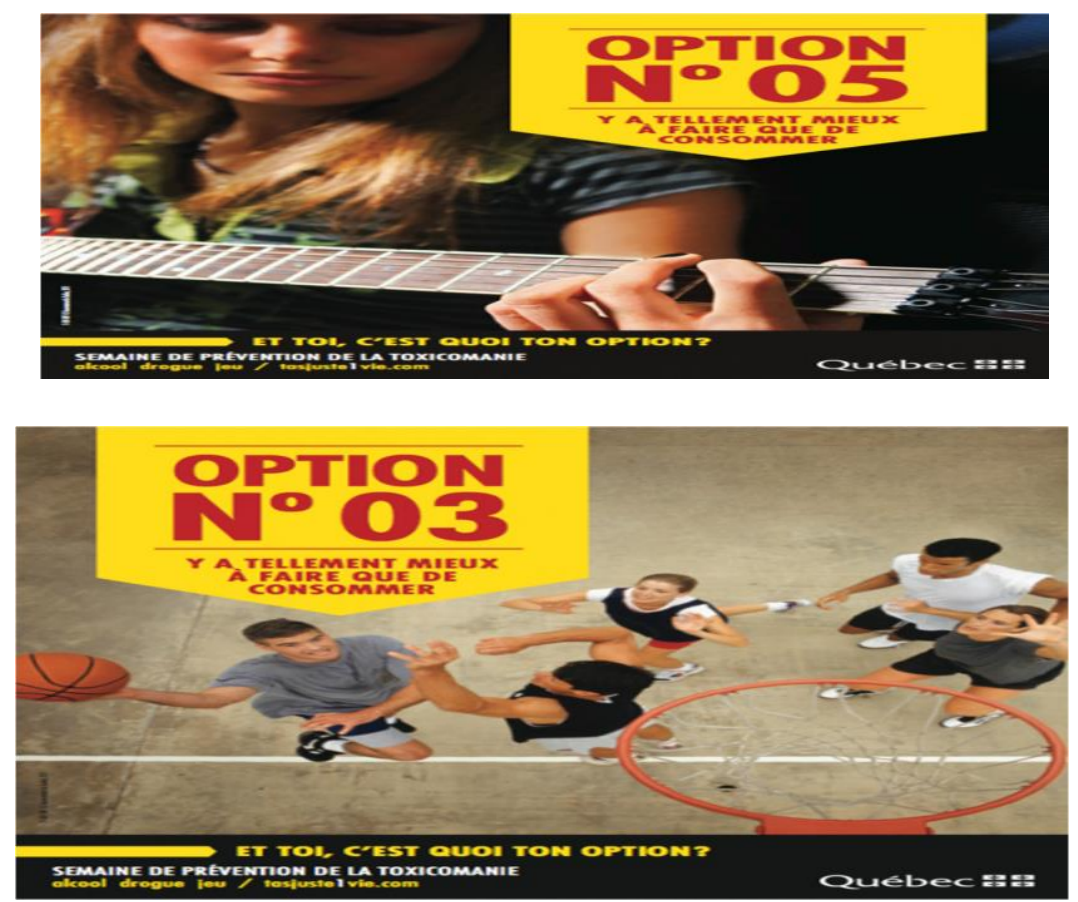

Ces deux affiches figurent parmi une série relative à la prévention de la toxicomanie, comme l'indique la légende « Semaine de prévention de la toxicomanie. »

Pour décoder le message, l'interprétation spontanée est probablement la plus simple et la plus évidente. II suffit d'associer le slogan à l'image pour aboutir à la conclusion suivante : Jouer de la guitare, ou du basketball vaut mieux que consommer de la drogue. 
Dans ce cas l'argument réside dans l'image elle-même, qui est devenu indispensable à l'affiche publicitaire. Le slogan « $Y$ a tellement mieux à faire que de consommer » revêt un aspect très général, mais une fois associé à l'image, il acquiert un effet beaucoup plus fort sur les consommateurs de drogue. Les images du sport ou de la musique figurent par suite comme des exemples sur les activités qui pourraient se substituer à la consommation des drogues.

Ainsi, l'argument produit un effet perlocutoire, car «faire du sport est mieux que de consommer » n'a pas été explicitement mentionnée, l'association de l'image au slogan a produit un effet sur le récepteur qui devrait changer son attitude et s'orienter vers des activités alternatives à la consommation.

Nous pouvons donc proposer pour ce slogan la traduction suivante :

\begin{tabular}{|c|c|}
\hline L.D & L.A \\
\hline $\begin{array}{l}\text { Y a tellement mieux à faire que } \\
\text { de consommer...Et toi, c'est } \\
\text { quoi ton option? }\end{array}$ & 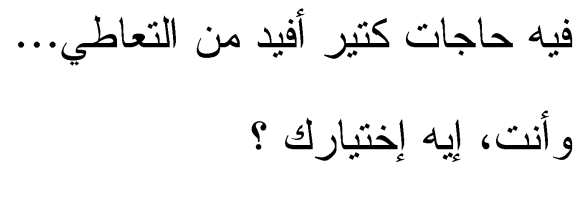 \\
\hline
\end{tabular}


Nous avons opté pour le langage familier, dans la langue d'arrivée, vu que la langue de départ a employé le même registre, avec l'ellipse du sujet «Y a », au lieu de «II y a ». De plus cette annonce vise essentiellement les jeunes. II serait donc plus adéquat d'employer un registre familier, d'autant plus que le texte source a privilégié le tutoiement, afin de toucher plus facilement les jeunes sans barrière, ce que nous avons rendu en arabe par « أنت " C'est en respectant ces éléments que l'on a effectivement pu atteindre l'objectif de cette annonce argumentative.

Mais si l'argument ou la rhétorique sont toujours secondés par l'image, en tant que support ou moteur de l'effet perlocutoire escompté, est-ce que l'image peut, à elle seule, servir d'axe central pour une publicité ? Peut-on trouver une publicité ou une affiche de sensibilisation dépendant uniquement de l'image sans la moindre inscription textuelle ? L'image peut-elle se passer du texte, de l'argument et de la rhétorique pour jouer, elle seule, tout le rôle escompté par la publicité ?

Nous allons nous pencher, dans les pages suivantes, sur le rôle de l'image publicitaire en tant que seul pivot de certaines campagnes de sensibilisation ou même de simples publicités, 
ainsi que sur les composantes essentielles qui contribuent largement à faire passer le message.

\section{Image publicitaire}

«L'image participe pleinement au dispositif stratégique de la publicité, à travers la mise en scène attractive du produit et l'impact qu'elle exerce consciemment ou non, sur ses récepteurs. " (Adam, 2005 :193)

II est donc évident que la publicité accorde une place de choix à la représentation iconique qui stimule les sens des récepteurs.

«L'image peut être légitimement considérée comme un texte au sens fort du texte dans la mesure où ses constituants et leur distribution dans l'espace de la représentation vont solliciter de la part du spectateur une série d'ajustement dont on peut dire qu'ils se ramènent à ce qu'on appelle précisément la lecture.» (Adam, $2005:$ 191)

Dans cette optique, nous pouvons voir que l'image joue un rôle fortement argumentatif dans la mesure où elle synthétise tout le message escompté, car elle focalise sur le sens ou sur l'effet voulu, et émet parfois un cri d'alarme contre certains dangers, ou 
certains phénomènes, ce qui est fréquent dans la plupart des campagnes de sensibilisation.

« L'image est effectivement destinée à plaire à son spectateur ou à lui procurer des sensations spécifiques, en faveur de l'idée véhiculée. » (Aumont, 1990 : 57)

L'image, dans ce sens, s'axe sur des données matérielles qui renvoient à des référents mondains, "reposant sur des techniques bien connues : cadrage, localisation, mise en plan, perspective, effet de profondeur et angle de vision. "(Adam, $2005: 196)$

Nous allons voir dans les affiches suivantes comment l'image peut, à elle seule, transmettre tout le message dans les publicités de promotion, aussi bien que dans les campagnes de sensibilisation, si elle exige une adaptation ou non.

Dans les affiches suivantes, nous verrons comment les campagnes de sensibilisation peuvent uniquement dépendre sur les images et leurs effets perlocutoires sur le public cible. Nous analyserons le sujet de l'affiche, son objectif ainsi que les indices de valorisation du message. 
L'affiche publicitaire suivante appartient au dentifrice Colgate :
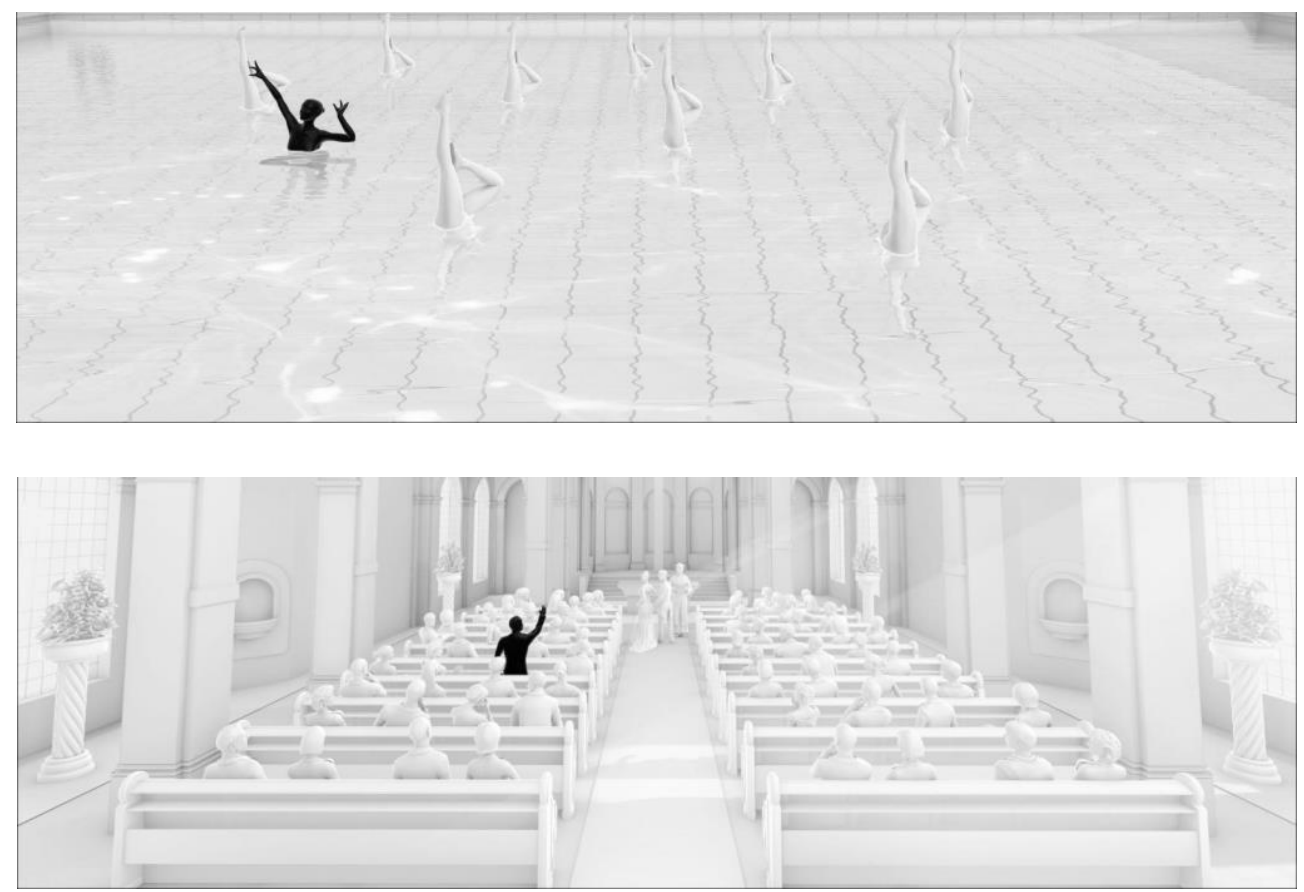

Nous pouvons voir comment cette publicité est considérablement révélatrice. Sans la moindre inscription textuelle, à part le logo de la marque qui figure en haut de l'affiche, ces deux affiches font la promotion du dentifrice Colgate. Avec uniquement deux couleurs prédominante, le noir et le blanc, cette photographie revêt tout le sens et le message que l'entreprise veut associer à Colgate.

Dans la première affiche, c'est un concert de ballet nautique et on voit que l'élément noir fait un mouvement intrus par rapport aux autres. Dans la seconde, nous voyons une 
cérémonie de mariage à l'église, tout est dans l'ordre, tout est cohérent, à part cet intrus qui est venu gâcher toute la scène en se levant en pleine récitation du serment de mariage. Une seule tâche noire, une seule carie peut nuire à tout le tableau. Le dentifrice Colgate va, en ce sens, camoufler toutes ces impuretés et rendre l'harmonie de la scène.

Voilà tout le message que veut transmettre l'entreprise à ses consommateurs, un message qui réside dans le contraste qu'évoque la confrontation du noir et du blanc. C'est comme si le dentifrice Colgate pouvait blanchir toute la scène. Cette publicité s'axe sur le cadrage de l'image qui focalise sur le contraste entre le noir et le blanc.

Évidemment si l'on veut transposer cette affiche pour un public arabe, rien ne changera, car la notion du noir et du blanc est universelle et n'exige aucune adaptation.

Voyons l'affiche suivante alarmante contre les effets de la déforestation et de l'intervention de l'homme dans la nature :

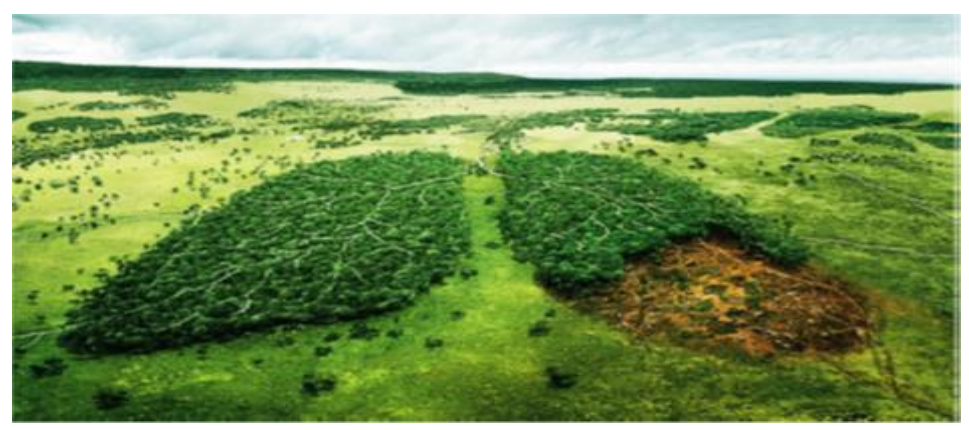


Nous pouvons déceler une sorte de métaphore résidant dans l'image, où l'illustrateur a assimilé la nature à des poumons et où l'intervention humaine est considérée comme un facteur ravageur de l'équilibre et de la sérénité de la nature. Nous voyons que cette affiche repose sur une perspective élargie et sur une vue panoramique de la scène, qui capte l'image d'en haut.

Dans notre conscience collective, la couleur verte représente le progrès, la sérénité, mais par-dessus tout, la prospérité, alors que le marron représente plutôt un effet d'amertume, de pollution, de poussière, et de dégoût.

Le contraste de ces deux couleurs révèle tout le message et nous alarme sur les répercussions de la déforestation sur la nature.

Passons maintenant à une affiche pour la sensibilisation contre les effets néfastes du tabac :

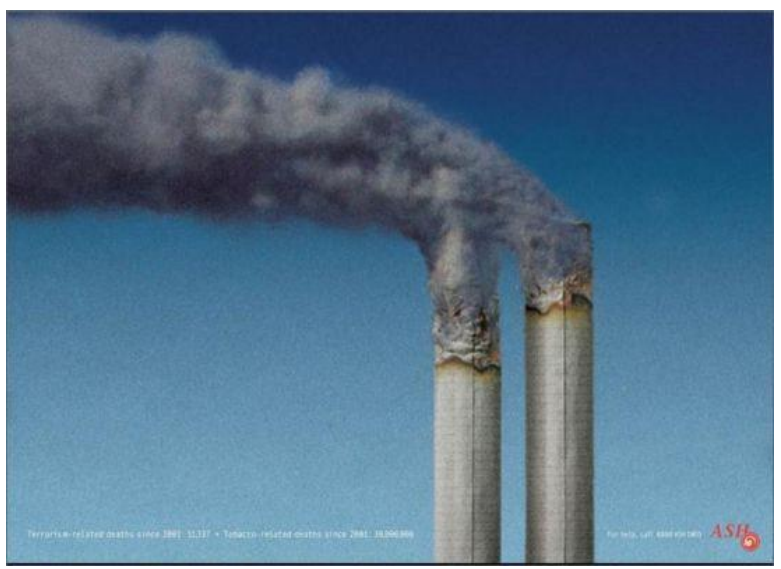


Cette affiche nous présente un cadrage focalisant sur les deux cigarettes et sur le contraste entre la couleur bleu, couleur de paix et de sérénité et la couleur grise, couleur du danger, de la pollution et de la dépression.

Cette affiche visant le tabac et les fumeurs nous sensibilise contre les dangers qui peuvent découler du tabac. II existe effectivement une métaphore évidente et très puissante sur cette affiche, qui provoque et engendre tous les effets voulus, et illustre toute la gravité du tabac et des dangers qui en résultent.

Le concepteur de cette image a effectivement eu recours à une astuce très pertinente, celle d'établir un parallélisme entre ces deux cigarettes et les cheminées des usines industrielles qui engendrent un grave taux de pollution. Comme ces usines mettent en péril notre santé, les cigarettes le font aussi, comme si le tabac pouvait engendrer des dangers similaires à ceux provoqués par la pollution industrielle.

C'est ainsi que l'image peut se substituer indépendamment au rôle de l'argumentation et de la rhétorique et à toute inscription textuelle, de manière à déclencher les réactions requises du public destinataire. 


\section{Conclusion}

Nous avons passé en revue des échantillons de publicités où la rhétorique prédomine, une publicité où l'adaptation est la méthode choisie par le traducteur, ainsi que des publicités où l'argument est tantôt explicite et tantôt implicite

Nous avons également vu que cet argument, qu'il soit direct ou indirect, doit garder son aspect tel qu'il est dans la traduction. Si l'argument est explicite, c'est parce que le texte est injonctif ou interrogatif, comme dans l'affiche du tabac. C'est pourquoi nous ne pouvons pas le rendre implicite dans la traduction, au contraire, il doit rester explicite. Dans ce cas c'est le texte qui régit notre traduction.

Nous avons également vu d'autres arguments implicites, comme ceux relatifs aux deux dernières affiches, celles destinées à la lutte contre la consommation des drogues. L'argument implicite tient au fait que l'argument réside dans l'association de l'image au slogan, raison pour laquelle, dans notre traduction, nous ne pouvons pas expliciter l'argument, ou ajouter une information à celle présentée sur l'affiche.

Au fil de cette étude, nous avons pu nous pencher de manière non exhaustive évidemment, sur les différentes difficultés qui peuvent se poser à un traducteur dans le domaine de la publicité. 
Nous avons pu voir comment les entreprises publicitaires mobilisent une panoplie d'outils afin d'attirer l'attention du public, de l'appeler à contribution pour déchiffrer le message, et de l'inciter à analyser les composantes de la publicité de manière plus ou moins spontanée. Ces entreprises cherchent effectivement à frapper l'esprit du public cible, à capter tous ses sens, à s'adapter à son bagage socioculturel, en somme, à l'impliquer dans le processus communicatif publicitaire.

Dans cette optique, et vu tous ces moyens explorés par ces entreprises, le traducteur publicitaire se trouve confronté à de nombreuses entraves d'ordre linguistique, rhétorique, communicatif et argumentatif. II se trouve obligé de surmonter tous ces défis, car c'est sur lui qu'incombe toute la responsabilité de communiquer le message publicitaire.

Le rôle du traducteur est extrêmement subtil et important, d'autant plus qu'il doit adapter la publicité à son destinataire, sans pourtant faillir au message source et à son effet perlocutoire.

En explorant ces difficultés, cachées derrière les coulisses, nous avons vu comment la rhétorique peut remarquablement être bénéfique pour la publicité, et comment elle peut non seulement résider dans le texte, mais également dans l'image iconique ellemême. C'est ainsi que le traducteur se trouve obligé de 
reproduire le même effet et la même créativité que confère la rhétorique au message publicitaire, en tant que technique de persuasion très efficace et très méticuleuse.

Nous avons également vu, que parmi les piliers sur lesquels s'axe la publicité, figure l'argumentation qui caractérise la publicité car elle s'adresse à la raison, et stimule la réflexion du récepteur, afin d'influencer son comportement vis-à-vis de la publicité pour le mener à adopter une certaine réaction.

Nous avons vu comment l'argumentation peut être narrative, en situant le public cible dans la situation, et comment elle peut être informative, en présentant des données renforçant l'idée en question.

D'autre part, loin de toute inscription textuelle, nous avons vu comment l'image constitue en elle-même un pivot essentiel de la publicité, un pivot qui peut, de manière indépendante, s'imposer comme l'unique canal par lequel véhicule tout le message. Ceci a été clairement démontré à travers les affiches des campagnes de sensibilisation, s'axant sur les couleurs, le cadrage, l'angle de vision et la perspective. Tous ces éléments sont fortement révélateurs dans la formation des affiches publicitaires. 
Parfois le message peut être révélé par la seule représentation iconique qui nécessite de la part du récepteur un travail de déchiffrement des éléments conjugués ensemble.

Nous avons donc, à travers notre étude analytique, pu constater que la traduction du texte publicitaire n'est pas aussi simple qu'elle ne le paraît ; à cela s'ajoute la nécessité que la publicité s'adapte au milieu socioculturel de ses récepteurs. II doit prendre en considération leur historique, leur savoir, aussi bien que leurs croyances et leurs attentes.

Au dénouement de ce travail, nous pouvons dire que le traducteur publicitaire est toujours face au dilemme de l'authenticité et de la fidélité traductionnelle, qui a effectivement fait couler beaucoup d'encre.

\section{Bibliographie}

\section{Corpus :}

Échantillons d'affiches publicitaires, extraits de l'internet.

II. Ouvrages sur la traduction publicitaire :

1. ADAM J-M., (2005) L'argumentation publicitaire, éd, Armand Colin, Paris.

2. AUMONT J., (1990) L'image, éd, Armand Colin, Paris.

3. BACRY P., (1992) Les figures de styles, éd, Belin, Paris.

4. CHUQUET H., \& PAILLARD M., (1987) Approches linguistiques des problèmes de traduction Anglais_Français, éd, Ophrys, Paris. 
5. FONTANIER P., (2004) Les figures du discours, éd, Flammarion, Paris.

6. GERVERAU L., (1994) Voir, comprendre, analyser les images, éd, Guides Repères, Paris.

7. GUIDÈRE M., (2000), Publicité et traduction, éd, L'Harmattan, paris.

8. GUIDÈRE M., (2008), La communication multilingue. Traduction commerciale et institutionnelle, Bruxelles.

9. GUIDERE M., (2010), Introduction à la traductologie, éd, De Boek, Paris.

10. LEDERER M., (1994), La traduction aujourd'hui, éd, Hachette, Paris.

11. KAPFERER J-N., (1998), Les chemins de la persuasion publicitaire, éd, Presses Universitaires de France, Paris.

III. Revues et périodiques :

1. BOIVINEAU R., (1972), L'A.B.C. de l'adaptation publicitaire, in Meta, V. 17, № 1, mars 1972, pp.5-28p.

2. BROULAND P., (2006), Rhétorique de la publicité dans le français contemporain, Acta Oeconomica Pragensia, roè. 14, è. 4, pp. 82-86.

3. CAPELLI S., (2007), Évaluer l'impact d'un visuel publicitaire métaphorique, In Actes du XXIIlème Congrès International de l'AFM 31 mai \& 1er juin, Aix-les-Bains, 32p.

4. DENIS M., (1974), «L'Express » : un style publicitaire, In:

Communication et langages. № 21, 1974. pp. 96-115.

5. DURAND J., (1970), Rhétorique et images publicitaire, Communications, № $15, \mathrm{pp} .70-95$.

6. GUIDÈRE M., (2011), Les corpus publicitaires : nouvelles approches et méthodes pour le traducteur, in : Meta : journal des traducteurs, vol. 56, № 2, pp. 336-350. 
7. Elisabet Å., (2011), Le problème de la traduction des métaphores, Linnaeus University, 33p.

8. ISABEL Cómitre Narváez., (2005), Traduction et non-traduction en contexte publicitaire : analyse contrastive des marques et slogans de l'Oréal (France-Espagne), in Parallèles, № 27(2), octobre 2015 pp 2955.

9. MICHEL C., (1968), Argumentation publicitaire et études de motivation. In Les Cahiers de la publicité, № 20, pp. 42-44.

10. TATILON C., (1990) Le texte publicitaire : traduction ou adaptation ? in Meta. V. 35, № 1, mars 1990, pp. 243-250.

IV. Dictionnaire

MORIER H., (1961) Dictionnaire de Poétique et de Rhétorique. éd, Presses Universitaires de France, Paris.

$\mathrm{V}$. Thèses et mémoires :

1.BREDUILLIEARD P., L'efficacité du message publicitaire à argumentation biréférentielle, 2009, 263p.

2. TISON J-B., Étude comparative de messages publicitaires anglophones et francophones à la lumière d'éléments culturels, University of North Texas, December 2012, 36p.

VI. Sitographie :

1. DANSEREAU, Stéphanie, "L'Image publicitaire », Uqam, mars 2004 http://www.er.uqam.ca/nobel/r33554/pub.html.

2. DURAND, Jacques, «Rhétorique et image publicitaire », Page perso, avril 2007

http://jacques.durand.pagesperso-orange.fr/Site/Textes/t9.htm. 
3. GUIDERE, Mathieu, article sur « De la traduction publicitaire à la communication multilingue », Revue Meta, septembre 2009 https://www.erudit.org/revue/meta/2009/v54/n3/038306ar.html?vue=re sume.

4. HERVE-BAZIN, Grégoire, « Les figures de rhétorique dans la communication », Le monde des études, septembre 2008 http://lemondedesetudes.fr/rhetoriques-dans-la-com-3/.

5. SJOFNAE, "Les figures de rhétorique dans la publicité », Lovecommunication, mars 2013

http://www.love-communication.fr/les-figures-de-rhetorique-dans-laublicitea87924881.

6. SJOFNAE, "Les types de discours et leurs intérêts dans la publicité », Lovecommunication, mars 2013

http://www.love-communication.fr/les-types-de-discours-et-leursinteret-dansla-publicite-a86614277.

7. SCOTT, David, "La rhétorique de l'image/ texte, dans l'affiche française Imageandnarrqtive, mars 2008

http://www.imageandnarrative.be/inarchive/affiche_contemporaine/scott .htm.

8. Académie des sciences commerciales http://academiedesciencescommerciales.org/dictionnaire_new/definition .php?id=5940 date de consultation 2015

9. http://www. pubenstock.com/2013/publicite-rhetorique-figures-destyle-langage-discours/date de consultation 2015

10. http://caseeworld.com/rentree/cours/download.php?f=b9d055148fda7d a3e4d70c5fcae3491c.pdf date de consultation 2015 
11. http://www.enseignons.be/upload/secondaire/francais/01-0210version-prof.doc date de consultation 2015

12. http://www.gredos.usal.es/jspui/.../1/DTI_LarminauxC_Traductionadaption.pdf.date de consultation 2015

13. http://www.love-communication.fr/les-figures-de-rhetorique-dans-lapublicite-a87924881.date de consultation 2015

14. http://www.pubenstock.com/2013/publicite-rhetorique-figures-destyle-langage-discours/date de consultation 2015

15. http://www.love-communication.fr/les-types-de-discours-et-leursinteret-dans-lapublicite-a86614277date de consultation 17-12-2015

16. https://www.larousse.fr/dictionnaires/francais/coupe-faim/19815 date de consultation 2020

17.https://www.almaany.com/ar/dict/ar/\%D8\%B2\%D9\%86\%D9\%82\%D8\% A9date de consultation 2020/

المر اجع العربية

1. فايد، وفاء (2007) معجم التعابير الإصطلاحية فى العربية المعاصرة ، دار النشر :

د.ن.، القاهرة.

2. عنانى، محمد (2000) فن الترجمة، دار النشر : لونجمان ، القاهرة.

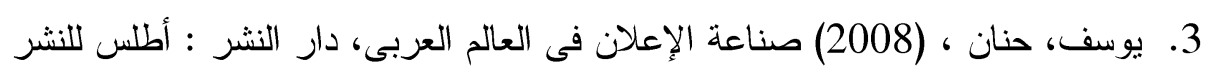

و الإنتاج الإعلامى، القاهرة.

4. تمام، حسان، (1973) اللغة العربية معناها ومبناها، الهيئة المصرية العامة للكتاب، القاهرة.

5. د. د. محمد أحمد منصور، (2001) النزجمة بين النظرية و التطبيق، دار الكمال ،دمشق.

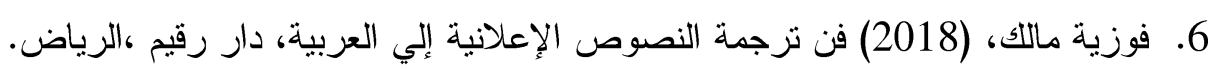

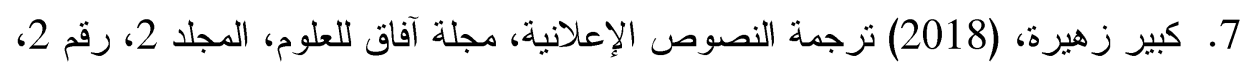

291-298 ص - مارس، الجز ائر. 